\title{
Health service utilization and access to medicines among Syrian refugee and host community children in Lebanon
}

\author{
Emily Lyles ${ }^{1}$, Baptiste Hanquart ${ }^{2}$, the LHAS Study Team, Michael Woodman ${ }^{3}$ and Shannon Doocy ${ }^{1 *}$
}

\begin{abstract}
Background: With over 500,000 Syrian refugee children in Lebanon, we undertook this study to assess unmet child health needs and health service utilization among Syrian refugees and affected host communities in Lebanon with the aim of informing humanitarian programming.

Methods: A cross-sectional survey of Syrian refugees and host communities in Lebanon was conducted using a two-stage cluster survey design with probability proportional to size sampling. The questionnaire focused on access to health services, including a module on care seeking for children.

Results: The care seeking rate was $74.2 \%$ among refugee and $89.0 \%$ among host community households with a child less than 18 years seeking medical care the last time it was needed. Refugee households most often sought care in primary health care centers (52.7\%), followed by pharmacies (22.2 \%) and private clinics (17.8\%), whereas host community households most often sought care in private clinics $(47.6 \%)$ and primary health care centers (23.2\%). Among child care seekers, $95.2 \%$ of refugee and $94.6 \%$ of host community children were prescribed medication during the most recent visit, of which 92.7 and $97.3 \%$, respectively, obtained the medication. Overall, $66.0 \%$ of refugee and $75.9 \%$ of host community households reported out-of-pocket expenditures for either the consultation or prescribed medications at the most recent visit (refugee mean US\$30.4; host community mean US\$56.0).

Conclusions: Care seeking was significantly lower among refugees than the host community. Out-of-pocket payments were considerable for both groups, the majority of which were for medication, and cost was the primary barrier to both care seeking and attaining medications.
\end{abstract}

Keywords: Syria, Lebanon, Refugee, Host community, Humanitarian assistance, Children, Health services

\section{Introduction}

The influx of refugees fleeing war in Syria since early 2011 is illustrative of a growing trend in forced displacement towards settlement in urban areas and middle-income countries (Spiegel et al. 2010; Gutierres and Spiegel 2012). Humanitarian assistance efforts have adapted to meet this shift, replacing traditional camp-based assistance strategies to integrated care that is better suited to meet the unique needs of non-camp refugees. With Lebanon now hosting the highest number of refugees per capita in the

\footnotetext{
* Correspondence: doocy1@jhu.edu

${ }^{1} J o h n s$ Hopkins Bloomberg School of Public Health, 615 N Wolfe St, Suite

E8132, Baltimore, MD 21205, USA

Full list of author information is available at the end of the article
}

world, the increased burden of providing care to more than 1.2 million Syrian refugees through the Lebanese health system carries immense costs for service delivery and strengthening health system capacity and infrastructure to meet the growing need (UNHCR 2015b). In contrast to other hosting countries in the region, Lebanon has not established formal refugee camps for Syrians (UNHCR 2015c). Assistance provided to Syrian refugees in Lebanon is based on a primary health care strategy wherein primary health care services are subsidized in existing health centers across the country and referrals for secondary and tertiary services are managed by a third-party private administrator (World Bank 2013; OCHA 2014; UNHCR 2013, 2015a). 
Among global displaced populations, the greatest mortality rates are found in children under 1 year and under 5 years of age (Toole and Waldman 1990, 1997; Guha-Sapir and Panhuis 2004). In the Syrian refugee context, children under the age of five account for $17.7 \%$ of the refugee population and those under age 17 account for approximately half (51.5\%) of the population (UNHCR 2015c). This distribution poses even greater challenges to host countries given that mortality causes and disease burdens of displaced populations are often similar to populations with high child mortality rates. Prevalence of communicable diseases among displaced populations may be comparatively higher than rates observed both among the host country population and prior to the conflict (Moss et al. 2006; WHO 2015). In light of the ongoing crisis in Syria and ever-growing challenges in meeting the unique health needs of refugees in host countries, we undertook this study to assess the access to and utilization of health services among Syrian refugee children in non-camp settings in Lebanon.

\section{Methods}

A survey of Syrian refugees and Lebanese host communities was conducted in March and April 2014 to characterize health-seeking behaviors and health service access. A cluster design with probability proportional to size sampling was used to attain a nationally representative sample of Syrian refugees living outside of camps. Sample size was determined for key objectives based on the most conservative prevalence estimate of $50 \%$; calculations assumed $80 \%$ power and a design effect of 2.0. The planned sample was increased from the minimum identified size of 900 refugee households to 1400 refugee households and 700 Lebanese host community households to provide increased precision of point estimates and additional power.

Given the concentration of Syrian refugees and the low cost of visiting many locations due to the country's small size, a 100 cluster $\times 21$ household (14 Syrian refugee households and seven host community households) design was used. Probability proportional to size sampling using UNHCR registration data was used to assign clusters to cadastrals, assuming that non-registered refugees had similar residence patterns. Permission to survey in certain security-sensitive areas as planned could not be attained which necessitated a re-draw of 28 clusters assigned to 22 inaccessible cadastrals. Clusters were re-assigned to accessible areas using probability proportional to size sampling. The final cluster assignment included 35 clusters $(35 \%)$ in the North governorate, 34 clusters (34\%) in Bekaa governorate, 25 clusters (25\%) in Mount Lebanon governorate, four clusters (4 \%) in Beirut governorate, and two clusters (2\%) in the South governorate (Fig. 1). Only two cadastrals in the South were accessible to the survey team; presenting data from only these locations was not sufficiently representative of the governorate and would violate cluster sampling assumptions, thus the two South governorate clusters were excluded from the analysis.

ARC GIS software was used to randomly allocate cluster start points within cadastrals. Coordinates in populated areas were used, and the nearest intersection to the start point, usually within a half kilometer, was used as the starting survey location. Teams were provided with coordinates, and satellite imagery maps, and were instructed to navigate to start points using mapping software such as Google Maps. At the start location, interviewer pairs were sent in different directions to locate households; they approached the nearest business likely to be used by refugees and asked to be referred to nearby Syrian households. Other key informants, notably community residents, were used when there were no nearby shops. When interviewers reached a household that consented to participate, the first interview in the cluster was conducted; upon completion, respondents were asked for a referral or introduction to the nearest Syrian household. This referral process was used until 14 Syrian refugee interviews were completed. Following each two completed interviews with Syrian refugee households, interviewers proceeded to the nearest Lebanese household and completed an interview. To improve representativeness and geographic coverage of the sample, no more than three households within the same apartment building were included.

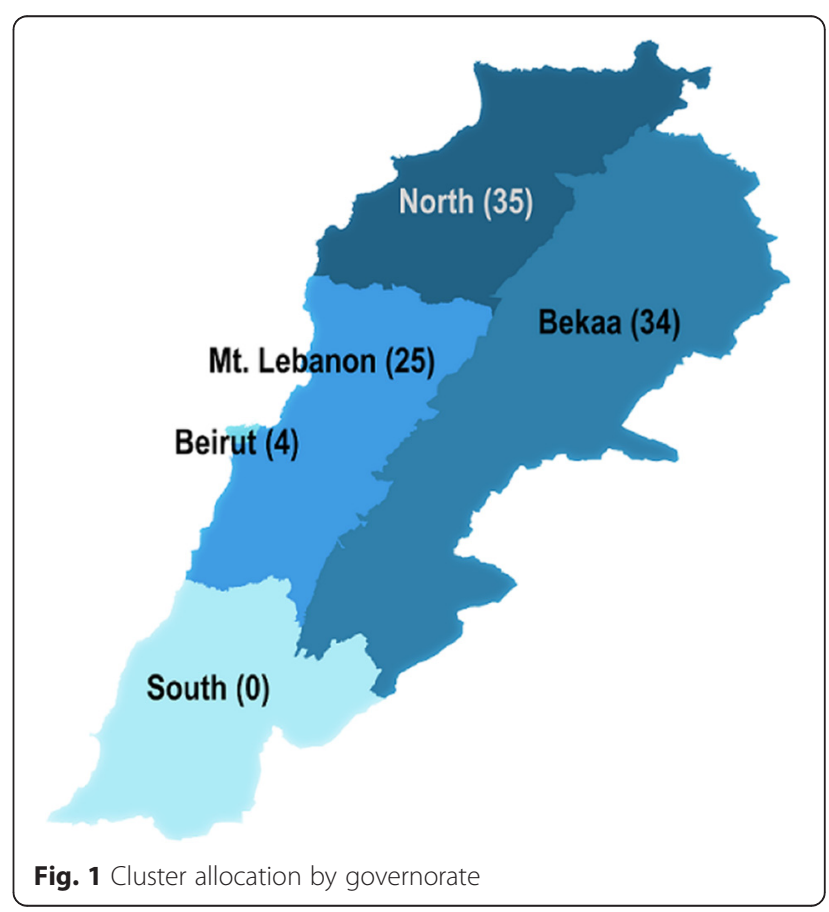


To sample informal tented settlements (ITSs), the team estimated the size and area by walking transects and/or the perimeter. When necessary, the ITS was divided into sub-areas of similar size that were assigned to different interviewers. Interviewers located the middle of the settlement/area, spun a pen to randomly select a direction, and then walked in the indicated direction counting the number of shelters passed before reaching the edge of the settlement/area. A randomly selected number between one and the total number of shelters passed was used to identify the starting household. This process was repeated until the necessary number of interviews was complete; referrals were not requested in ITSs to reduce the potential for bias.

Only Syrian households arriving in Lebanon in 2011 or later were eligible to participate, as the aim was to capture the experiences of those displaced by the conflict; however, only one household arrived in Lebanon before 2011. Families with both Lebanese and Syrian members were considered Syrian refugees if they arrived in Lebanon in 2011 or later and lived in Syria prior to this time; families who had never lived in Syria were considered as Lebanese host community households for the survey.

The questionnaire was initially developed for use in Jordan and was adapted to the Lebanese context by consensus between partner organizations. The questionnaire focused on health service utilization, access and barriers to care, children's health, and chronic medical conditions. The Arabic translation of the Jordan questionnaire was adapted for Lebanon and a formal pilot test conducted. Interviewers received 2 days of classroom training that focused on the questionnaire, e-data collection, interview techniques, basic principles of human subjects' protections, and sampling after which two additional days of practical field training were held. To protect the anonymity of respondents, no unique identifiers were recorded and verbal consent was obtained. Interviews lasted between 30 and 60 min depending on the household size, number of children, and individuals with chronic medical conditions. Data was collected on tablets using the Magpi mobile data platform by DataDyne LLC (Washington, DC). Data was analyzed using Stata 13 (College Station, TX) and Tableau Desktop (Seattle, WA) using descriptive statistics and standard methods for comparison of means and proportions. Differences in household characteristics by care seeking in Lebanon were examined using chi-square and $t$ test methods. Characteristics with statistical significance $p<0.10$ were included in the adjusted logistic regression model. The Stata 'svy' command was used to account for the cluster survey design so that standard errors of the point estimates and model coefficients were adjusted for survey design effects.
The study was approved by the Institutional Review Board at American University of Beirut. The Johns Hopkins Bloomberg School of Public Health Institutional Review Board also reviewed the protocol and determined that members of the JHSPH team were not involved in human subjects research because they did not have direct contact with participants or access to personal identifiers.

\section{Results}

A total of 2165 households were approached to participate. Of these, $1.9 \%(n=40)$ were not at home, $0.2 \%$ $(n=4)$ were previously interviewed, $0.05 \%(n=1)$ was ineligible, and $2.7 \%(n=58)$ refused. The final sample included 2062 households (1376 Syrian refugee and 686 host Lebanese households), which equates to a response rate of $93.6 \%$. Overall, $66.6 \%$ of refugee households and $28.1 \%$ of host community households had at least one child $0-5$ years of age; $69.0 \%$ of refugee households and $48.5 \%$ of host community households had at least one child 6-17 years of age. Children age $0-5$ years and 6-17 years accounted for 31.0 and $32.0 \%$, respectively, of the refugee survey population and 9.5 and $22.1 \%$ of the host community survey population, respectively.

\section{Care seeking and health service utilization}

Respondents were asked to report on the most recent time a child in their household, age 17 years or younger, needed medical care (Table 1). A sizeable proportion of both host community (62.4\%) and Syrian refugee households $(70.0 \%)$ reported a child needing medical care within the month preceding the survey. Care seeking was higher in the host community where $87.3 \%$ of households reported medical attention was sought the last time a child needed care as compared to $79.4 \%$ of Syrian refugee households $(p<0.001)$; there were no significant differences in the care seeking rate by region in either population ( $p=0.616$ and $p=0.412$, respectively). Among the $25.7 \%$ of Syrian refugee and $11 \%$ of host community households that did not receive care for a child the last time it was needed, the primary reason was cost: $92 \%$ of refugee households and $69.2 \%$ of host community households reported they could not afford to seek medical services for the child. The most frequently reported other reason for not seeking care among both refugee and host community households was that the family deciding care was not needed (2.4 and $17.9 \%$, respectively).

Conditions for which care was sought were similar in both groups and are as follows: respiratory illness (60.8 \% of refugees and $66.4 \%$ of the host community), fever $(8.4 \%$ of refugees and $4.6 \%$ of the host community), diarrhea ( $3.6 \%$ of refugees and $9.2 \%$ of the host community), skin problems (6.0 \% of refugees and $3.1 \%$ 
Table 1 Health care seeking in Lebanon among Syrian refugees and Lebanese host community children

\begin{tabular}{|c|c|c|c|c|c|}
\hline & \multicolumn{2}{|c|}{ Syrian refugees } & \multicolumn{2}{|c|}{ Host community } & \multirow{2}{*}{$\begin{array}{l}\text { Population } \\
\text { comparison } \\
p \text { value }\end{array}$} \\
\hline & $\%$ & {$[95 \mathrm{Cl}]$} & $\%$ & {$[95 \mathrm{Cl}]$} & \\
\hline Last time care was needed $^{a}$ & \multicolumn{2}{|c|}{$n=1144$} & \multicolumn{2}{|c|}{$n=378$} & \\
\hline Less than 2 weeks ago & 45.5 & {$[41.9,49.1]$} & 35.2 & {$[29.9,40.8]$} & \multirow[t]{6}{*}{$<0.001$} \\
\hline 2 weeks to $<1$ month ago & 29.5 & {$[26.4,32.7]$} & 32.8 & {$[28.5,37.5]$} & \\
\hline 1 month to $<3$ months ago & 17.3 & {$[14.9,20.0]$} & 19.6 & {$[15.4,24.5]$} & \\
\hline 3 months to $<6$ months ago & 4.4 & {$[3.3,5.8]$} & 4.5 & {$[2.8,7.1]$} & \\
\hline 6 months to $<1$ year ago & 1.6 & {$[1.0,2.4]$} & 1.6 & {$[0.6,4.0]$} & \\
\hline More than 1 year ago & 1.8 & {$[1.1,3.0]$} & 6.3 & {$[4.1,9.7]$} & \\
\hline Reason for most recent care needed ${ }^{a}$ & \multicolumn{2}{|c|}{$n=1123$} & \multicolumn{2}{|c|}{$n=354$} & \\
\hline Respiratory problem & 61.1 & {$[58.1,64.0]$} & 60.5 & {$[55.9,64.9]$} & \multirow[t]{12}{*}{0.371} \\
\hline Fever & 8.1 & {$[6.6,10.0]$} & 7.6 & {$[5.2,11.1]$} & \\
\hline Diarrhea & 5.0 & {$[3.8,6.6]$} & 8.5 & {$[6.2,11.6]$} & \\
\hline Skin problem & 3.9 & {$[2.9,5.2]$} & 2.3 & {$[1.1,4.4]$} & \\
\hline Injury & 3.7 & {$[2.7,4.9]$} & 4.0 & {$[2.2,7.0]$} & \\
\hline Ear problem & 3.2 & {$[2.3,4.4]$} & 3.7 & {$[2.2,6.2]$} & \\
\hline Asthma & 3.3 & {$[2.4,4.6]$} & 1.1 & {$[0.4,3.0]$} & \\
\hline Dental care & 2.6 & {$[1.8,3.7]$} & 4.2 & {$[2.6,6.9]$} & \\
\hline Eye problem & 1.5 & {$[0.9,2.4]$} & 2.0 & {$[1.0,4.0]$} & \\
\hline Behavioral/emotional problem & 0.8 & {$[0.4,1.6]$} & 0.3 & {$[0.0,2.0]$} & \\
\hline Worms & 0.3 & {$[0.1,0.8]$} & 0 & & \\
\hline Other & 6.6 & {$[5.2,8.3]$} & 5.9 & {$[3.9,8.9]$} & \\
\hline Received attention last time care was needed ${ }^{a}$ & \multicolumn{2}{|c|}{$n=1123$} & \multicolumn{2}{|c|}{$n=354$} & \\
\hline Yes, sought and received care & 74.2 & {$[70.2,77.8]$} & 89.0 & {$[84.9,92.0]$} & \multirow[t]{3}{*}{$<0.001$} \\
\hline Did not seek care & 18.6 & {$[15.8,21.8]$} & 8.5 & {$[5.9,11.9]$} & \\
\hline Sought, but did not receive care & 7.1 & {$[5.4,9.4]$} & 2.5 & {$[1.2,5.4]$} & \\
\hline \multicolumn{6}{|l|}{ Location of most recent care in Lebanon $^{b}$} \\
\hline Primary health care center & 52.7 & {$[48.1,57.2]$} & 23.2 & {$[18.1,29.1]$} & \multirow[t]{5}{*}{$<0.001$} \\
\hline Private clinic (cabinet) & 17.8 & {$[15.0,21.0]$} & 47.6 & {$[41.8,53.5]$} & \\
\hline Hospital & 5.6 & {$[4.1,7.6]$} & 12.7 & {$[9.3,17.1]$} & \\
\hline Pharmacy & 22.2 & {$[18.7,26.2]$} & 14.9 & {$[11.3,19.5]$} & \\
\hline Other & 1.7 & {$[0.8,3.6]$} & 1.6 & {$[0.6,4.5]$} & \\
\hline \multicolumn{6}{|l|}{ Facility type of most recent care ${ }^{b}$} \\
\hline Primary/secondary/private provider & 70.8 & {$[66.7,74.7]$} & 71.1 & {$[65.7,76.0]$} & \multirow[t]{3}{*}{$<0.001$} \\
\hline Hospital & 5.6 & {$[4.1,7.6]$} & 12.7 & {$[9.3,17.1]$} & \\
\hline Pharmacy or shop & 23.5 & {$[19.8,27.7]$} & 16.2 & {$[12.3,21.0]$} & \\
\hline Reason for not receiving needed care ${ }^{c}$ & \multicolumn{2}{|c|}{$n=289$} & \multicolumn{2}{|c|}{$n=39$} & \\
\hline Could not afford provider costs & 92.0 & {$[87.1,95.2]$} & 69.2 & {$[54.5,80.9]$} & \multirow[t]{7}{*}{$<0.001$} \\
\hline Family decided care should not be sought & 2.4 & {$[1.1,5.5]$} & 17.9 & {$[9.0,32.6]$} & \\
\hline No transportation/difficult to access & 1.0 & {$[0.1,7.2]$} & 2.6 & {$[0.4,16.0]$} & \\
\hline Could not afford transportation costs & 1.0 & {$[0.3,3.2]$} & 0 & & \\
\hline Disliked long wait time on previous visit(s) & 1.0 & {$[0.3,3.3]$} & 0 & & \\
\hline Did not know where to go & 0.7 & {$[0.2,2.7]$} & 0 & & \\
\hline Appointment scheduled/still waiting & 0.3 & {$[0.0,2.5]$} & 2.6 & {$[0.4,16.0]$} & \\
\hline
\end{tabular}


Table 1 Health care seeking in Lebanon among Syrian refugees and Lebanese host community children (Continued)

\begin{tabular}{llll}
\hline Provider's equipment or drugs are inadequate & 0.3 & {$[0.0,2.5]$} & 0 \\
Could not take time off work/other commitments & 0 & & 2.6 \\
Security & 0.3 & {$[0.0,2.5]$} & 0 \\
Other & & \\
\hline as percent of all household index cases that needed care in Lebanon & \\
b As percent of all household index cases that received care in Lebanon \\
cAs percent of all household index cases that did not receive needed care in Lebanon
\end{tabular}

of the host community), and injury (3.9\% of refugees and $3.1 \%$ of the host community) ( $p=0.371)$. The conditions for which care was sought were similar across the regions (refugee $p=0.792$; host community $p=0.475$ ) but differed significantly by facility type $(p<0.001$ for both groups) (Fig. 2).

Location of most recent care in Lebanon differed significantly between refugee and host community households. Among refugee households that sought care for a sick child, approximately half ( $53.0 \%)$ went to a primary health care center (Table 1), $22.6 \%$ sought care at pharmacies, $16.0 \%$ at private clinics, and $5.4 \%$ at hospitals. A significantly higher proportion of host community care seekers received care at private clinics $(45.0 \%)$, followed by primary health care centers $(27.5 \%)$, pharmacies (17.6\%), and hospitals (8.4\%). No statistically significant differences in care seeking location were observed by region in either population (refugee $p=0.086$; host community $p=0.452$ ).

Among child care seekers, a similar proportion of refugee and host community care seekers (95.2 and $94.6 \%$, respectively) were prescribed medication during the most recent visit (Table 2); no significant differences were observed by region in either group $(p=0.962$ and $p=0.974$, respectively). The proportion of host community children receiving a prescription did not vary significantly by sector type $(p=0.222)$; however, significant differences were observed among refugee children as follows: primary health care centers, $96.4 \%$; private clinics, $93.2 \%$; and hospitals, $83.0 \%(p<0.001)$. Of those prescribed medications, a significantly greater proportion of host community households $(97.3 \%)$ were able to obtain all prescribed medicines as compared to $92.7 \%$ of refugee households $(p=0.007)$.

\section{Spending on child health}

Of the 1144 refugee and 378 host community families with sick children needing care identified in the survey, 833 (74.2\%) refugee households and 315 (89\%) host community households sought care or treatment for their child. Among these families, some incurred out-ofpocket costs and some did not. The average total cost per episode of illness among all 833 refugee families who sought care was $\$$ US30.4 (US\$12 for consultations,
US $\$ 18.4$ for medications). The median cost per illness episode among refugee child care seekers, however, was US\$19.9. No statistically significant differences in costs to refugees were observed by region $(p=0.199)$; however, total cost per illness episode varied significantly by type of facility where care was sought (US\$77.2 for hospitals compared to US\$48.1 for private clinics, and US\$25.5 at primary health care centers; $p<0.001$ ). Among the 315 host community child health care seekers, the average total cost per care episode was US\$56 (US $\$ 25.5$ for consultations and US\$30.5 for medications) and the median total cost was US\$41.1. As was observed in refugee care seekers, no statistically significant differences in costs to host communities were observed by region $(p=0.075)$; however, differences across facility types were statistically significant (US\$126.2 for hospitals, US\$57.5 for private clinics, and US $\$ 37.7$ for primary health care centers; $p<0.001)$. Details about the total and component costs of treatment (consultations and medications) are provided in Tables 3 and 4 and Fig. 3 .

Among the 833 refugee families who sought care or treatment for their sick child, 92.4\% $(n=770)$ reported any out-of-pocket payment (paying for either consultation, medication, or both, Table 3). Among only these refugee families who paid for consultations and/or medications, the average total payment was \$US32.9 (US\$ 13.0 for consultations, US\$19.9 for medications). The median values of these payments were as follows: total cost, US\$23.2; consultation cost, US\$3.3; and medication cost, US\$15.9. There were no statistically significant differences in costs across the three regions among these 770 refugee families $(p=0.176)$, but mean payment amount varied across facility types as follows: hospitals, US\$86.4; private clinics, US\$48.4; and primary health care centers, US\$27.9 $(p<0.001)$. Among only the 303 host community families reporting any payment for consultations and/or medications, the average total payment was US\$58.2 (US\$26.5 for consultations and US\$31.7 for medications) and the median total costs was US $\$ 43.1$. There were no statistically significant differences in costs by region among these 303 families $(p=0.057)$, but costs varied across facility types as follows: hospitals, US $\$ 129.4$; private clinics, US $\$ 58.7$; and primary health care centers, US\$39.9 $(p<0.001)$. 

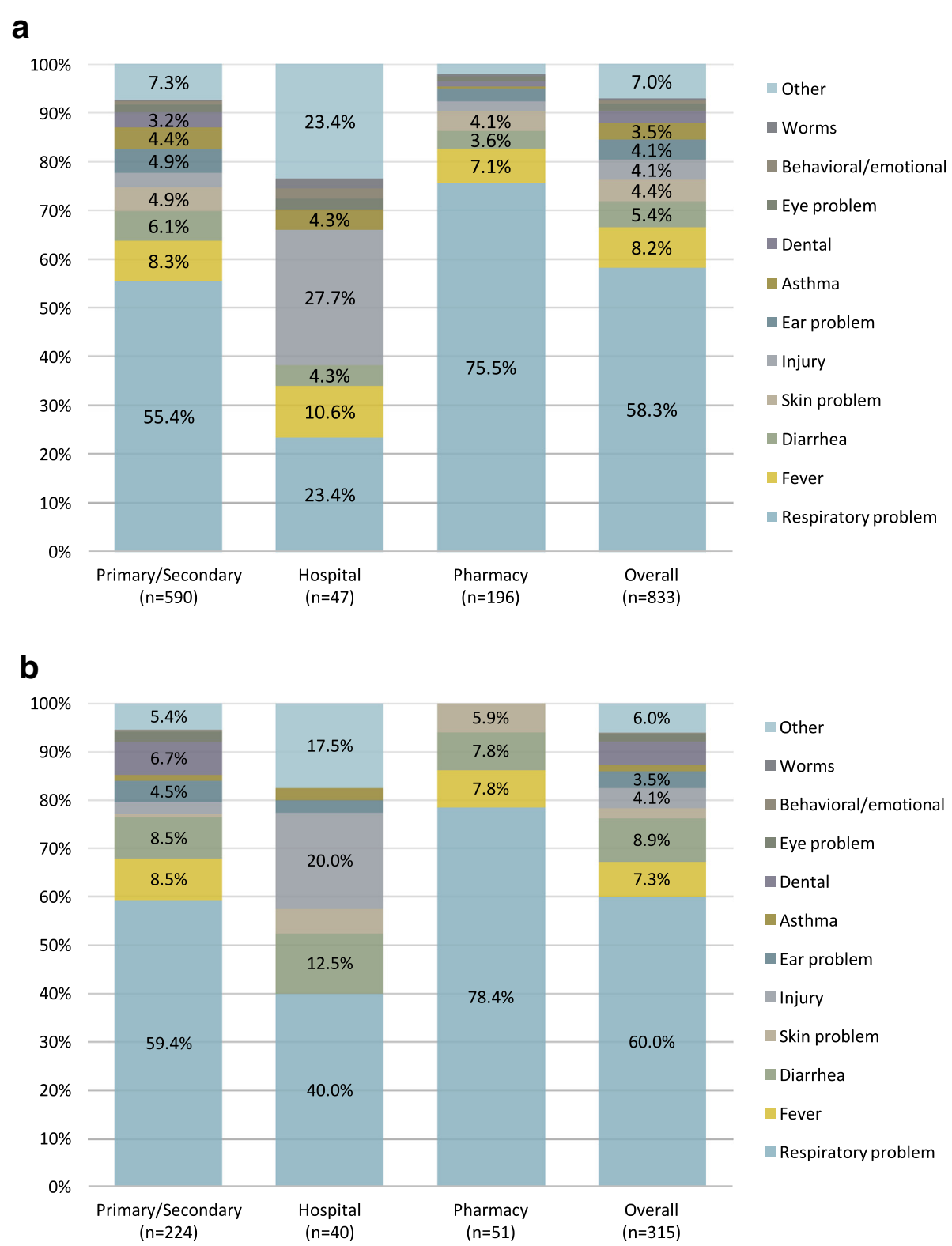

Fig 2 a Reasons for child care seeking by sector among Syrian refugees in Lebanon. b Reasons for child care seeking by sector among host communities

Among the 1144 refugee families who sought care or treatment for their sick child, 550 reported paying for consultation, regardless of whether or not they paid for medications (Table 3). Among these refugee families who paid for consultations, the average and median cost per consultation was US\$18.1 and US\$6.6, respectively. There were statistically significant differences across regions $(p=0.049)$ and types of facilities $(p<0.001)$ in the average consultation costs paid by these 550 refugee families. The average consultation cost was US\$21.6 for refugees settled in Bekaa, US\$19.9 for refugees settled in Beirut/Mount Lebanon, and US\$14 for refugees settled in the North. The average consultation cost to refugees was US $\$ 70.3$ at hospitals, US $\$ 25.7$ at private clinics, and US\$9.9 at primary health care centers.

Among the 378 host community families who sought care or treatment for their sick child, 239 reported paying for consultation, regardless of whether or not they paid for medications (Table 4). Among these host community families who paid for consultations, the average and median cost per consultation was US $\$ 33.6$ and US $\$ 26.5$, respectively. There were statistically significant differences across regions $(p=0.032)$ and types of facilities $(p<0.001)$ in the average consultation costs paid by these 239 host community families. The average consultation cost was 
Table 2 Medication access among Syrian refugees and host community children in Lebanon

\begin{tabular}{|c|c|c|c|c|c|c|c|c|c|}
\hline & \multirow{2}{*}{\multicolumn{2}{|c|}{ Survey total }} & \multicolumn{7}{|c|}{ By region } \\
\hline & & & \multicolumn{2}{|c|}{ Beirut/Mt. Lebanon } & \multicolumn{2}{|l|}{ Bekaa } & \multicolumn{2}{|c|}{ North } & \multirow[t]{2}{*}{ Regional comparison $p$ value } \\
\hline & $\%$ & {$[95 \mathrm{Cl}]$} & $\%$ & {$[95 \mathrm{Cl}]$} & $\%$ & {$[95 \mathrm{Cl}]$} & $\%$ & {$[95 \mathrm{Cl}]$} & \\
\hline \multicolumn{10}{|c|}{ Prescribed medication during most recent health facility visit } \\
\hline \multirow[t]{2}{*}{ Syrian refugees } & \multicolumn{2}{|c|}{$n=833$} & \multicolumn{2}{|c|}{$n=234$} & \multicolumn{2}{|c|}{$n=267$} & \multicolumn{2}{|c|}{$n=332$} & \\
\hline & 95.2 & {$[93.5,96.5]$} & 94.9 & {$[91.7,96.9]$} & 95.5 & {$[91.4,97.7]$} & 95.2 & {$[92.3,97.0]$} & 0.974 \\
\hline \multirow[t]{2}{*}{ Host community } & \multicolumn{2}{|c|}{$n=315$} & \multicolumn{2}{|l|}{$n=86$} & \multicolumn{2}{|l|}{$n=98$} & \multicolumn{2}{|c|}{$n=131$} & \\
\hline & 94.6 & {$[91.6,96.6]$} & 94.2 & {$[87.8,97.3]$} & 94.9 & {$[87.0,98.1]$} & 94.7 & {$[89.7,97.3]$} & 0.962 \\
\hline Population comparison $p$ value & 0.691 & & 0.772 & & 0.845 & & 0.825 & & \\
\hline \multicolumn{10}{|l|}{ By facility type ${ }^{a}$} \\
\hline \multicolumn{10}{|l|}{ Primary health care center } \\
\hline Syrian refugees & 96.4 & {$[94.1,97.8]$} & 96.3 & {$[91.1,98.5]$} & 96.8 & {$[91.3,98.8]$} & 96.0 & {$[92.3,98.0]$} & 0.932 \\
\hline Host community & 97.3 & {$[89.7,99.3]$} & 93.3 & {$[66.7,99.0]$} & 100 & & 97.2 & {$[82.6,99.6]$} & 0.465 \\
\hline Population comparison $p$ value & 0.685 & & 0.510 & & 0.443 & & 0.738 & & \\
\hline \multicolumn{10}{|l|}{ Private clinic } \\
\hline Syrian refugees & 93.2 & {$[87.7,96.4]$} & 93.6 & {$[83.0,97.8]$} & 100 & & 86.8 & {$[75.3,93.4]$} & 0.025 \\
\hline Host community & 92.7 & {$[87.6,95.8]$} & 93.6 & {$[82.7,97.8]$} & 90.9 & {$[79.1,96.3]$} & 93.2 & {$[84.1,97.3]$} & 0.849 \\
\hline Population comparison $p$ value & 0.848 & & 1.000 & & 0.029 & & 0.252 & & \\
\hline \multicolumn{10}{|l|}{ Hospital } \\
\hline Syrian refugees & 83 & {$[70.3,90.9]$} & 66.7 & {$[38.3,86.6]$} & 82.4 & {$[59.3,93.7]$} & 94.4 & {$[72.8,99.1]$} & 0.113 \\
\hline Host community & 97.5 & {$[84.4,99.6]$} & 100 & & 94.1 & {$[69.4,99.1]$} & 100 & & 0.529 \\
\hline Population comparison $p$ value & 0.031 & & 0.037 & & 0.307 & & 0.358 & & \\
\hline \multicolumn{10}{|c|}{ Able to obtain all medications prescribed at most recent visit ${ }^{\mathrm{b}}$} \\
\hline \multirow[t]{2}{*}{ Syrian refugees } & \multicolumn{2}{|c|}{$n=784$} & \multicolumn{2}{|c|}{$n=220$} & $n=25$ & & $n=31$ & & \\
\hline & 92.7 & {$[90.4,94.5]$} & 92.3 & {$[86.9,95.5]$} & 92.9 & {$[88.3,95.7]$} & 92.9 & {$[89.2,95.5]$} & 0.962 \\
\hline Host community & $n=2$ & & $n=81$ & & $n=25$ & & $n=12$ & & \\
\hline & 97.3 & {$[94.8,98.6]$} & 100 & & 95.7 & {$[89.2,98.3]$} & 96.8 & {$[92.2,98.7]$} & 0.183 \\
\hline Population comparison $p$ value & 0.007 & & 0.043 & & 0.378 & & 0.140 & & \\
\hline Reason for not obtaining medications ${ }^{c}$ & & & & & & & & & \\
\hline Syrian refugees & $n=5$ & & $n=17$ & & $n=18$ & & $n=22$ & & \\
\hline Could not afford the medication & 86.0 & {$[73.1,93.3]$} & 94.1 & {$[69.2,99.1]$} & 77.8 & {$[57.1,90.2]$} & 86.4 & {$[56.2,96.9]$} & 0.589 \\
\hline Medication out of stock at facility & 12.3 & {$[5.5,25.3]$} & 5.9 & {$[0.9,30.8]$} & 16.7 & {$[5.9,38.9]$} & 13.6 & {$[3.1,43.8]$} & \\
\hline Symptoms improved & 1.8 & {$[0.2,11.5]$} & 0 & & 5.6 & {$[0.8,29.5]$} & 0 & & \\
\hline Other & 0 & & 0 & & 0 & & 0 & & \\
\hline Host community & $n=8$ & & $n=0$ & & $n=4$ & & $n=4$ & & \\
\hline Could not afford the medication & 87.5 & {$[45.3,98.3]$} & Insuffic & cient sample s & size for $r$ & regional and & oopulat & & \\
\hline Medication out of stock at facility & 0 & & & & & & & & \\
\hline Symptoms improved & 0 & & & & & & & & \\
\hline Other & 12.5 & {$[1.7,54.7]$} & & & & & & & \\
\hline
\end{tabular}

${ }^{a}$ As percent of household index cases that received care at this facility type

${ }^{\mathrm{b}}$ As percent of household index cases prescribed medication

${ }^{\mathrm{C}}$ As percent of household index cases that did not obtain prescribed medication

US\$40.8 for those settled in Bekaa, US\$36.3 for those settled in Beirut/Mount Lebanon, and US\$26.1 for those settled in the North. The average consultation cost to host community child care seekers was US\$86.5 at hospitals,
US $\$ 29.3$ at private clinics, and US\$11.7 at primary health care centers.

Among the 1144 refugee families who sought care or treatment for their sick child, 667 reported paying for 
Table 3 Out-of-pocket payments for consultation fees, medications, and health care visit in Lebanon among Syrian refugees (all costs presented in USD)

\begin{tabular}{|c|c|c|c|c|c|c|c|c|c|c|c|c|c|c|c|c|c|}
\hline & & \multirow{2}{*}{\multicolumn{2}{|c|}{ Survey total }} & \multicolumn{7}{|c|}{ By region } & \multicolumn{7}{|c|}{ By facility type } \\
\hline & & & & \multicolumn{2}{|c|}{ Beirut/Mt. Lebanon } & \multicolumn{2}{|l|}{ Bekaa } & \multicolumn{2}{|c|}{ North } & \multirow{2}{*}{$\begin{array}{l}p \\
\text { value }\end{array}$} & \multicolumn{2}{|c|}{ PHCC } & \multicolumn{2}{|c|}{ Private clinic } & \multicolumn{2}{|c|}{ Hospital } & \multirow{2}{*}{$\begin{array}{l}p \\
\text { value }\end{array}$} \\
\hline & & Point & $95 \mathrm{Cl}$ & Point & $95 \mathrm{Cl}$ & Point & $95 \mathrm{Cl}$ & Point & $95 \mathrm{Cl}$ & & Point & $95 \mathrm{Cl}$ & Point & $95 \mathrm{Cl}$ & Point & $95 \mathrm{Cl}$ & \\
\hline \multicolumn{2}{|l|}{ Among all care seekers } & \multicolumn{2}{|c|}{$n=833$} & \multicolumn{2}{|c|}{$n=234$} & \multicolumn{2}{|c|}{$n=267$} & \multicolumn{2}{|c|}{$n=332$} & & \multicolumn{2}{|c|}{$n=439$} & \multicolumn{2}{|c|}{$n=148$} & \multicolumn{2}{|l|}{$n=47$} & \\
\hline \multirow[t]{2}{*}{ Total costs } & Median & 19.9 & & 22.6 & & 21.9 & & 18 & & & 18.6 & & 43.1 & & 66.3 & & \\
\hline & Mean & 30.4 & {$[27.5,33.3]$} & 30.4 & {$[25.4,35.3]$} & 34.9 & {$[29.1,40.6]$} & 26.9 & {$[22.9,30.8]$} & 0.199 & 25.5 & {$[22.6,28.5]$} & 48.1 & {$[42.6,53.5]$} & 77.2 & {$[57.7,96.7]$} & $<0.001$ \\
\hline \multirow[t]{2}{*}{ Consultation costs } & Median & 3.3 & & 3.6 & & 3.3 & & 3 & & & 3.3 & & 19.9 & & 43.1 & & \\
\hline & Mean & 12 & {$[10.0,14.0]$} & 12.9 & {$[9.0,16.7]$} & 14.7 & {$[10.8,18.7]$} & 9.2 & {$[6.6,11.7]$} & 0.079 & 8.3 & {$[6.5,10.1]$} & 24.3 & {$[20.8,27.8]$} & 56.8 & {$[40.6,73.0]$} & $<0.001$ \\
\hline \multirow[t]{2}{*}{ Medication costs } & Median & 13.3 & & 13.3 & & 15.3 & & 13.3 & & & 13.3 & & 19.9 & & 17.9 & & \\
\hline & Mean & 18.4 & {$[17.0,19.9]$} & 17.5 & {$[15.0,20.0]$} & 20.1 & {$[17.4,22.8]$} & 17.7 & {$[15.4,20.0]$} & 0.967 & 17.2 & {$[15.3,19.1]$} & 23.8 & {$[20.1,27.5]$} & 20.4 & {$[13.6,27.2]$} & 0.016 \\
\hline \multicolumn{2}{|c|}{ Among care seekers with any payment } & \multicolumn{2}{|c|}{$n=770$} & \multicolumn{2}{|c|}{$n=221$} & \multicolumn{2}{|c|}{$n=236$} & \multicolumn{2}{|c|}{$n=313$} & & \multicolumn{2}{|c|}{$n=402$} & \multicolumn{2}{|c|}{$n=147$} & $n=42$ & & \\
\hline Total costs & Median & 23.2 & & 23.2 & & 25.2 & & 20 & & & 19.9 & & 43.1 & & 76.3 & & \\
\hline & Mean & 32.9 & {$[29.8,36.0]$} & 32.2 & {$[27.0,37.3]$} & 39.4 & {$[33.1,45.8]$} & 28.5 & {$[24.5,32.5]$} & 0.176 & 27.9 & {$[24.6,31.1]$} & 48.4 & {$[42.9,53.9]$} & 86.4 & {$[66.0,106.9]$} & $<0.001$ \\
\hline Consultation costs & Median & 3.3 & & 5.3 & & 4.6 & & 3.3 & & & 3.3 & & 19.9 & & 61.4 & & \\
\hline & Mean & 13 & {$[10.8,15.2]$} & 13.6 & {$[9.6,17.6]$} & 16.7 & {$[12.3,21.1]$} & 9.7 & {$[7.0,12.4]$} & 0.074 & 9.1 & {$[7.1,11.0]$} & 24.5 & {$[20.9,28.0]$} & 63.6 & {$[47.0,80.2]$} & $<0.001$ \\
\hline Medication costs & Median & 15.9 & & 15.9 & & 16.6 & & 13.3 & & & 13.3 & & 19.9 & & 19.9 & & \\
\hline & Mean & 19.9 & {$[18.4,21.5]$} & 18.5 & {$[16.0,21.1]$} & 22.8 & {$[19.8,25.7]$} & 18.8 & {$[16.5,21.1]$} & 0.918 & 18.8 & {$[16.7,20.9]$} & 23.9 & {$[20.2,27.6]$} & 22.8 & {$[15.0,30.7]$} & 0.031 \\
\hline Among households pay & or consultation & $n=550$ & & $n=151$ & & $n=18$ & & $n=21$ & & & $n=37$ & & $n=14$ & & $n=38$ & & \\
\hline Consultation costs & Median & 6.6 & & 10 & & 7.3 & & 6.6 & & & 4.6 & & 19.9 & & 66.3 & & \\
\hline & Mean & 18.1 & {$[15.3,21.0]$} & 19.9 & {$[14.8,25.1]$} & 21.6 & {$[16.3,27.0]$} & 14 & {$[10.1,17.9]$} & 0.049 & 9.9 & {$[7.8,11.9]$} & 25.7 & {$[22.0,29.4]$} & 70.3 & {$[53.5,87.1]$} & $<0.001$ \\
\hline Among households pay & or medications & $n=667$ & & $n=194$ & & $n=20$ & & $n=26$ & & & $n=33$ & & $n=12$ & & $n=30$ & & \\
\hline Medication costs & Median & 16.6 & & 16.6 & & 19.9 & & 16.6 & & & 16.6 & & 23.2 & & 28.2 & & \\
\hline & Mean & 23.0 & {$[21.2,24.8]$} & 21.1 & {$[18.2,24.1]$} & 26.2 & {$[22.8,29.6]$} & 22.0 & {$[19.2,24.7]$} & 0.861 & 22.8 & {$[20.3,25.3]$} & 28.2 & {$[24.5,31.8]$} & 32.0 & {$[22.8,41.2]$} & 0.006 \\
\hline
\end{tabular}


Table 4 Out-of-pocket payments for consultation fees, medications, and health care visit in Lebanon among host Lebanese (all costs presented in USD)

\begin{tabular}{|c|c|c|c|c|c|c|c|c|c|c|c|c|c|c|c|c|c|}
\hline & & \multirow{2}{*}{\multicolumn{2}{|c|}{ Survey total }} & \multicolumn{7}{|c|}{ By region } & \multicolumn{7}{|c|}{ By facility type } \\
\hline & & & & \multicolumn{2}{|c|}{ Beirut/Mt. Lebanon } & \multicolumn{2}{|l|}{ Bekaa } & \multicolumn{2}{|l|}{ North } & \multirow{2}{*}{$\begin{array}{l}p \\
\text { value }\end{array}$} & \multicolumn{2}{|l|}{$\mathrm{PHCC}$} & \multicolumn{2}{|c|}{ Private clinic } & \multicolumn{2}{|c|}{ Hospital } & \multirow{2}{*}{$\begin{array}{l}p \\
\text { value }\end{array}$} \\
\hline & & Point & $95 \mathrm{Cl}$ & Point & $95 \mathrm{Cl}$ & Point & $95 \mathrm{Cl}$ & Point & $95 \mathrm{Cl}$ & & Point & $95 \mathrm{Cl}$ & Point & $95 \mathrm{Cl}$ & Point & $95 \mathrm{Cl}$ & \\
\hline \multicolumn{2}{|l|}{ Among all care seekers } & \multicolumn{2}{|c|}{$n=315$} & \multicolumn{2}{|l|}{$n=86$} & \multicolumn{2}{|l|}{$n=98$} & \multicolumn{2}{|c|}{$n=131$} & & \multicolumn{2}{|l|}{$n=73$} & \multicolumn{2}{|c|}{$n=150$} & \multicolumn{2}{|l|}{$n=40$} & \\
\hline \multirow[t]{2}{*}{ Total costs } & Median & 41.1 & & 47.1 & & 44.8 & & 33 & & & 26.5 & & 49.8 & & 99.5 & & \\
\hline & Mean & 56.0 & {$[49.5,62.6]$} & 60.7 & {$[45.6,75.8]$} & 63.6 & {$[50.7,76.4]$} & 47.3 & {$[40.6,54.1]$} & 0.075 & 37.7 & {$[27.9,47.4]$} & 57.5 & {$[51.7,63.3]$} & 126.2 & {$[97.4,155.0]$} & $<0.001$ \\
\hline \multirow[t]{2}{*}{ Consultation costs } & Median & 16.6 & & 21.6 & & 19.9 & & 10 & & & 6.6 & & 26.5 & & 58 & & \\
\hline & Mean & 25.5 & {$[21.3,29.7]$} & 28.7 & {$[20.7,36.7]$} & 31.2 & {$[22.1,40.4]$} & 19.1 & {$[14.7,23.5]$} & 0.022 & 10.0 & {$[8.0,12.0]$} & 27.0 & {$[23.6,30.4]$} & 80.0 & {$[60.0,100.1]$} & $<0.001$ \\
\hline \multirow[t]{2}{*}{ Medication costs } & Median & 21.2 & & 23.2 & & 22.2 & & 19.9 & & & 16.6 & & 23.2 & & 33.2 & & \\
\hline & Mean & 30.5 & {$[26.4,34.6]$} & 32.0 & {$[22.9,41.1]$} & 32.3 & {$[25.3,39.3]$} & 28.2 & {$[22.5,34.0]$} & 0.449 & 27.7 & {$[18.4,37.0]$} & 30.5 & {$[25.5,35.6]$} & 46.2 & {$[29.4,62.9]$} & 0.078 \\
\hline \multicolumn{2}{|c|}{ Among care seekers with any payment } & \multicolumn{2}{|c|}{$n=303$} & \multicolumn{2}{|l|}{$n=82$} & \multicolumn{2}{|l|}{$n=95$} & \multicolumn{2}{|c|}{$n=126$} & & \multicolumn{2}{|l|}{$n=69$} & \multicolumn{2}{|c|}{$n=147$} & $n=39$ & & \\
\hline Total costs & Median & 43.1 & & 49.8 & & 46.4 & & 37 & & & 26.5 & & 49.8 & & 99.5 & & \\
\hline & Mean & 58.2 & {$[51.5,64.9]$} & 63.6 & {$[48.7,78.6]$} & 65.6 & {$[52.2,78.9]$} & 49.2 & {$[42.1,56.3]$} & 0.057 & 39.9 & {$[29.7,50.0]$} & 58.7 & {$[52.9,64.4]$} & 129.4 & {$[100.7,158.2]$} & $<0.001$ \\
\hline Consultation costs & Median & 19.9 & & 26.5 & & 19.9 & & 11.6 & & & 6.6 & & 26.5 & & 66.3 & & \\
\hline & Mean & 26.5 & {$[22.1,30.9]$} & 30.1 & {$[22.1,38.0]$} & 32.2 & {$[22.7,41.8]$} & 19.9 & {$[15.0,24.8]$} & 0.019 & 10.6 & {$[8.4,12.7]$} & 27.5 & {$[24.1,31.0]$} & 82.1 & {$[62.0,102.2]$} & $<0.001$ \\
\hline Medication costs & Median & 23.2 & & 23.2 & & 23.2 & & 20.6 & & & 16.6 & & 23.2 & & 33.2 & & \\
\hline & Mean & 31.7 & {$[27.7,35.8]$} & 33.6 & {$[24.4,42.7]$} & 33.3 & {$[26.3,40.4]$} & 29.4 & {$[23.7,35.1]$} & 0.404 & 29.3 & {$[19.7,38.9]$} & 31.2 & {$[26.1,36.3]$} & 47.4 & {$[30.4,64.3]$} & 0.097 \\
\hline Among households pay & or consultation & $n=23$ & & $n=68$ & & $n=75$ & & $n=96$ & & & $n=62$ & & $n=138$ & & $n=37$ & & \\
\hline Consultation costs & Median & 26.5 & & 33.2 & & 33.2 & & 19.9 & & & 8.3 & & 26.5 & & 66.3 & & \\
\hline & Mean & 33.6 & {$[28.5,38.7]$} & 36.3 & {$[28.2,44.4]$} & 40.8 & {$[29.5,52.1]$} & 26.1 & {$[19.9,32.2]$} & 0.032 & 11.7 & {$[9.6,13.9]$} & 29.3 & {$[25.9,32.8]$} & 86.5 & {$[66.4,106.6]$} & $<0.001$ \\
\hline Among households pay & or medications & $n=28$ & & $n=80$ & & $n=85$ & & $n=11$ & & & $n=80$ & & $n=85$ & & $n=37$ & & \\
\hline Medication costs & Median & 25.9 & & 23.2 & & 26.5 & & 23.2 & & & 19.9 & & 26.5 & & 33.2 & & \\
\hline & Mean & 34.1 & {$[29.6,38.6]$} & 34.4 & {$[25.0,43.8]$} & 37.2 & {$[28.8,45.7]$} & 31.6 & {$[25.5,37.7]$} & 0.559 & 31.6 & {$[21.4,41.8]$} & 34.4 & {$[29.0,39.9]$} & 49.9 & {$[32.6,67.2]$} & 0.093 \\
\hline
\end{tabular}




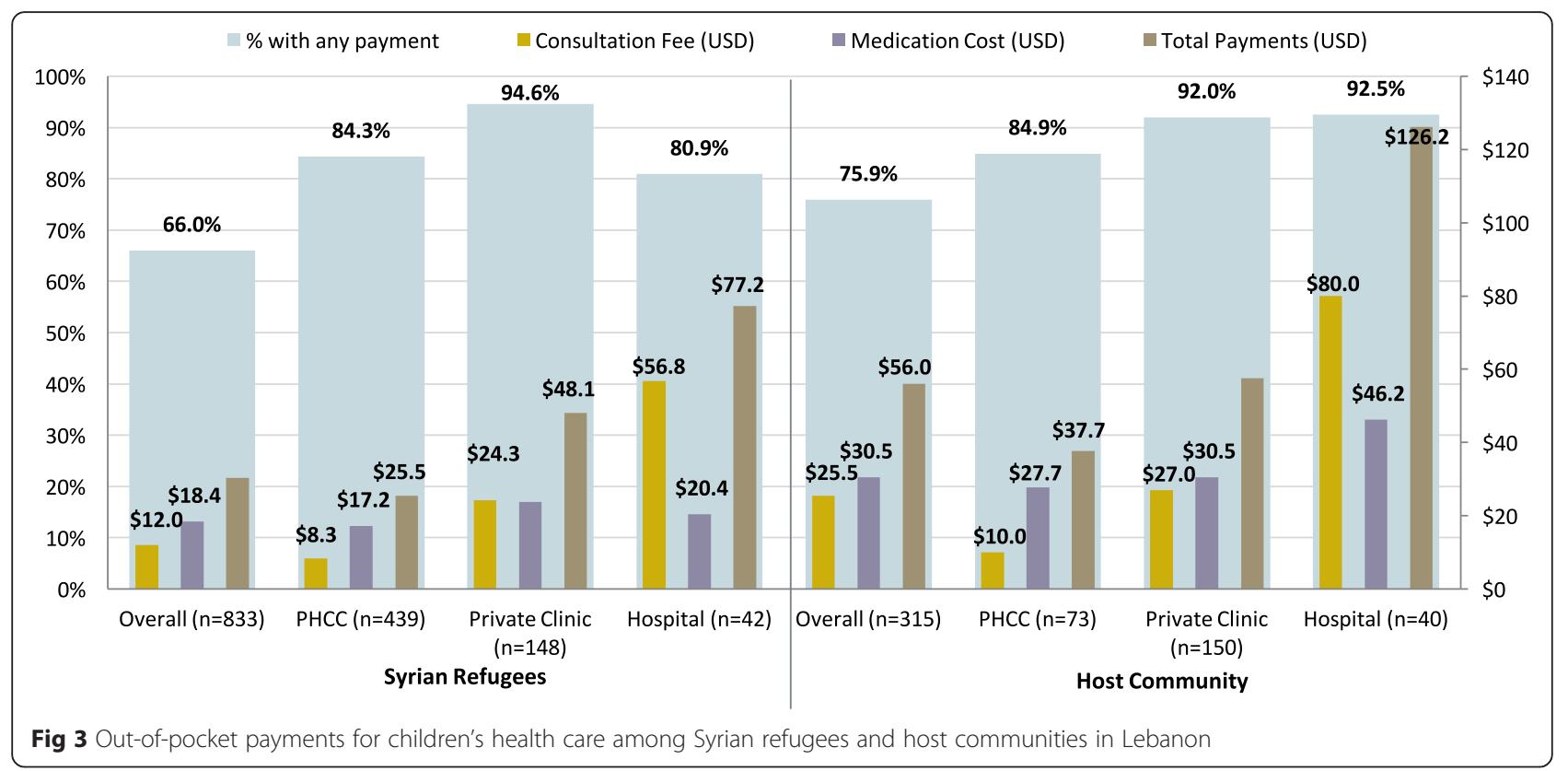

medications, regardless of whether or not they paid for a consultation (Table 3). Among these refugee families who paid for medications, the average cost per medication was $\$ 23.0$ (median $=\$ 16.6$ ). The differences in the average cost per medication (among refugee families who reported paying for medications) were not statistically significant across regions $(p=0.861)$ but did vary significantly across the three types of health facilities $(p=0.006)$ as follows: US $\$ 32.0$ at hospitals, US $\$ 28.2$ at private clinics, and US $\$ 22.8$ at primary health care centers. Among the 378 host community families who sought care or treatment for their sick child, 282 reported paying for medications, regardless of whether or not they paid for a consultation (Table 4). Among host community refugee families who paid for medications, the average cost per medication was \$34.1 (median $=\$ 25.9)$. The differences in the average cost per medication (among host community families who reported paying for medications) were not statistically significant across regions $(p=0.559)$ or across the three types of health facilities $(p=0.093)$.

\section{Discussion}

The average number of 4.16 health care visits per refugee child per year, estimated based on reported care seeking among the refugee survey sample, is slightly higher than the SPHERE standard recommendation of 2 to 4 visits and indicates good access to care for sick children among Syrian refugees in Lebanon. Extrapolating from survey findings, we estimate that the increased burden on the Lebanese health system from sick child visits among Syrian refugees alone is more than $1,800,000$ health visits per year including over 900,000 in the primary health care centers and over 350,000 in private clinics (Fig. 4). Given limited recent data on the Lebanese population and the fact that our sample of Lebanese households is representative of affected host communities and not the entire Lebanese population, extrapolation of the burden of Lebanese care visits would not provide reliable figures.

The most frequently reported cause of childhood illness among both Syrian refugees and host communities in Lebanon was respiratory problems, followed by fever, diarrhea, and skin problems. In light of this, it is possible that a number of care visits are not for severe illness. Additional community outreach and education that focuses on appropriate homecare options and decision making on when to seek care for minor illnesses could alleviate some of the caseload presenting at primary care facilities.

Injury was also a common reason for seeking care accounting for $4.1 \%$ of consultations in both refugee and host community populations. Among those presenting to hospitals for care, $27.7 \%$ of refugee and $20.0 \%$ of host community consultations were for injuries. Previous studies have found both burns and falls to be common among conflict-affected populations and refugees (O'Carrol 2015; Lafta et al. 2015). Given the poor living conditions of most refugees in Lebanon, particularly those in informal tented settlements, a similar pattern of unintentional injuries occurring in and around the home is likely. More information should be sought to better understand the causes of childhood injuries in this population in order to design appropriate interventions.

Both refugee and host community households reported relatively good access to needed child health care in Lebanon; however, health care was less accessible for 


\begin{tabular}{|c|c|c|}
\hline \multirow{2}{*}{\multicolumn{2}{|c|}{$\begin{array}{l}\text { 4.16 Average \# of visits per child } \\
2,305,365 \text { Total annual visits } \\
\end{array}$}} & \multirow{3}{*}{\begin{tabular}{|rl} 
By Careseeking Reason \\
$1,344,028$ & Respiratory Problem \\
189,040 & Fever
\end{tabular}} \\
\hline & & \\
\hline By Age Grot & up & \\
\hline $1,150,377$ & $0-4$ years & 124,490 Diarrhea \\
\hline 689,304 & 5-9 years & 101,436 Skin Problems \\
\hline 465,684 & $6-17$ years & 94,520 Injury \\
\hline \multicolumn{2}{|c|}{ By Location of Care } & 451,852 Others \\
\hline $1,214,928$ & Primary Health Care Center & By Facility Type \\
\hline 410,355 & Private Clinic (Cabinet) & 1,632,199 Primary/Secondary \\
\hline 64,421 & Hospital & 129,100 Hospital \\
\hline 255,384 & Pharmacy & 541,761 Pharmacy/Shop \\
\hline 360,278 & Other & 2,305 Others \\
\hline
\end{tabular}

Fig 4 Annual health service utilization projections for Syrian refugee children in Lebanon*

Syrian refugees than for the Lebanese host community. Overall, approximately one quarter of refugee children did not receive needed care or medicines, rates which were significantly higher than those observed for the Lebanese host community (11\%). Of these children not receiving needed care, a small proportion sought but did not receive care, accounting for $18.6 \%$ of refugee and $8.5 \%$ of host community children reporting needing care in Lebanon.

As with those that did not seek care the last time it was needed, the majority of households that sought but did not receive child health services cited cost as the barrier. More than half $(66.0 \%)$ of refugee families and nearly $80 \%$ of host community families with sick children report paying either consultation or medication costs at an average cost of about US $\$ 30.4$ and US $\$ 56.0$ per illness episode, respectively. Out-of-pocket payments averaged US\$33 and US\$59, respectively, among refugee and host community families who reported incurring any payment. Given current funding shortfalls, it is essential to address the question of whether or not the observed level of health care access will remain for refugees and the Lebanese host community. Health promotion programs aimed at improving health-seeking behavior and knowledge of home treatment for non-severe conditions such as upper respiratory infections may aid in decreasing the burden faced by families in caring for sick children.

A sizeable proportion (22.2\%) of refugee care seekers received care directly at pharmacies rather than first seeking consultation with primary or secondary health provider. This may be an indication that refugees are attempting to bypass facility care in order to lower outof-pocket expenses by avoiding payment of consultation fees. Significant differences in reason for needing care by facility type $(p=0.009)$ indicate different patterns in facility utilization by health concern among refugees. The most notable difference in reason for care was observed among those receiving care directly at pharmacies where $75.5 \%$ sought care for respiratory problems and $7.1 \%$ sought care for fever. This differed from primary/secondary providers and hospitals where only 55.4 and $23.4 \%$ of care was received for respiratory problems, respectively. Presumably, many of the respiratory problem and fever cases that presented at pharmacies were not perceived as severe and could be managed at home, a distinction which is important for both refugees in terms of reduced out-of-pocket payments and the health system because of the lower consultation burden when minor illnesses are managed at home.

Compared to other facility types, hospital utilization among refugees was primarily for injuries $(27.7 \%)$ with smaller proportions of care received for respiratory problems $(23.4 \%)$ and a host of other health problems including renal problems $(6.5 \%)$ and blood problems such as anemia $(6.5 \%)$. Such trends indicate a relatively high level of rational care seeking, indicating greater proportions of patients seeking care for conditions requiring complex treatments at more advanced facility types such as hospitals while less severe illnesses are predominantly treated using primary level care centers or pharmacies. Out-of-pocket costs to care seekers in private clinics and hospitals are significantly greater than primary health care centers and pharmacies, owing to the lack of consultation costs at pharmacies and subsidized care provided for refugees at primary health care centers. As such, more rational care seeking at primary health care centers or pharmacies could reduce out-ofpocket spending and, in turn, financial strain on refugee and affect host community households.

\section{Limitations}

With respect to sampling, reliance on UNHCR registration data may have resulted in sampling bias if the geographic distribution of registered and unregistered 
households differed. Reallocation of clusters in areas controlled by militarily and political factions where permission to conduct the survey was not secured, specifically in the South, Southern suburbs of Beirut, and northern areas of Bekaa, resulted in large area of the country being excluded. The survey coverage area included only $53 \%$ of registered Syrian refugees and thus is not representative of the entire Syrian refugee population in Lebanon. The within-cluster referral process presents the potential for bias, as respondents may not have always referred to the nearest household; referral procedures and small clusters size may have attenuated within-cluster similarities and the associated design effect. Replacement sampling, which was done for logistical purposes, could have contributed to bias if there were systematic differences between households with no one home compared with those interviewed. Additionally, the Lebanese host community sample was selected using a neighborhood approach and is reflective of those communities hosting the greatest number of refugees. As such, findings on the Lebanese host community population should not be generalized to the Lebanese population. Finally, interviews were conducted by Lebanese, which could have resulted in a higher refusal rate or influenced refugee responses to certain questions such as income.

\section{Conclusions}

Care seeking for Syrian refugee children was significantly lower than for Lebanese children in affected Lebanese host communities. Out-of-pocket payments were considerable for both groups, with medication costs averaging more than consultation fees, and cost was the primary reason for not seeking care and not attaining prescribed medications. While average out-of-pocket payments were lower for refugees than the host community, they are a sizeable expense given the limited income and humanitarian assistance received by most refugee households. The concern with high out-of-pocket payments is expected to persist given current shortfalls in humanitarian assistance funding and gradual declines in financial resources of refugee households that occur over extended periods of displacement. Training for providers on rational prescribing behavior and use of generic medicines could be an effective and low cost approach to reducing out-of-pocket expenditures while maintaining quality of care.

Given the size of the refugee population in Lebanon, this level of health service utilization has resulted in an immense burden on the health system, particularly in primary health care centers which are where refugees most often seek care for children (El-Jardali et al 2014; Refaat and Mohanna 2013). Public health education activities emphasizing home management of illness and self-care could help to better rationalize care seeking, reduce out-of-pocket expenditures, and reduce unnecessary visits to health facilities. Ongoing and expanded support to the primary health care system is required to reduce user fees, the cost of essential medications, and ensure adequate accessibility to and quality of care for both refugees and the Lebanese host community.

\section{Abbreviations}

ITS, informal tented settlement; UNHCR, United Nations High Commissioner for Refugees

\section{Competing interests}

The authors declare that they have no competing interests.

\section{Authors' contributions}

SD designed the study with support from the study team; EL and $\mathrm{BH}$ led the survey implementation and data collection with support from MW and the study team; EL led the data analysis and preparation of the manuscript with support from SD; MW contributed to interpretation of study findings and participated with selected members of the study team, in critical review of the manuscript. All authors read and approved the final manuscript.

\section{Acknowledgements}

The Lebanon Health Access Study Team includes Lara Chela, Nour Aridi, Nour Kassab, and Aline Keyrouz from Medecins du Monde; Abla Sibai and Fouad

Fouad from the Faculty of Health Sciences at American University of Beirut, and Deena Al-Shatti and Francois de La Roche from International Medical Corps.

\section{Author details}

'Johns Hopkins Bloomberg School of Public Health, 615 N Wolfe St, Suite E8132, Baltimore, MD 21205, USA. ${ }^{2}$ Medecins du Monde, Beirut, Lebanon.

${ }^{3}$ United Nations High Commissioner for Refugees, Beirut, Lebanon.

Received: 26 January 2016 Accepted: 25 May 2016

Published online: 16 July 2016

\section{References}

El-Jardali F, Hammoud R, Fouad FM, Bou Karoum L (2014) K2P briefing note: promoting access to essential health care services for Syrian refugees in Lebanon. Knowledge to Policy (K2P) Center, Beirut, https://www.aub.edu.lb/k2p/ policydialogue/Documents/K2P\%20Briefing\%20Note_Syrian\%20Refugees.pdf

Guha-Sapir D, Panhuis WG (2004) Conflict-related mortality: an analysis of 37 datasets. Disasters 28:418-428

Gutierres A, Spiegel P (2012) The state of the world's refugees: adapting health responses to urban environments. JAMA 308(7):673-674

Lafta R, Al-Shatari S, Cherewick M, Galway L, Mock C, Hagopian A, Flaxman A Takaro T, Greer A, Kushner A, Burnham G (2015) Injuries, death, and disability associated with 11 years of conflict in Baghdad, Iraq: a randomized household cluster survey. PLoS One 10(8):e0131834

Moss WJ, Ramakrishnan M, Storms D, Henderson Siegle A, Weiss W, Lenjnev I, Muhe $L$ (2006) Child health in complex emergencies. Bull World Health Organ 84(1):58-64

O'Carrol L (2015) Donate cash if you want to help Syrian refugees, aid groups say. The Guardian. http://www.theguardian.com/world/2015/sep/08/donatecash-help-syrian-refugees-aid-groups-unicef-wfp-say. Accessed 8 Dec 2015

Refaat MM, Mohanna K (2013) Syrian refugees in Lebanon: facts and solutions. The Lancet 382(9894):763-764

Spiegel P, Public Health, HIV Section at UNHCR, (2010) Urban refugee health: meeting the challenges. Forced Migr Rev 34:22

Toole MJ, Waldman RJ (1990) Prevention of excess mortality in refugee and displaced populations in developing countries. JAMA 263:3296-3302

Toole MJ, Waldman RJ (1997) The public health aspects of complex emergencies and refugee situations. Annu Rev Public Health 18:283-312

United Nations High Commissioner for Refugees (UNHCR) (2013) Health operational guidance, Lebanon., http://data.unhcr.org/syrianrefugees/ download.php?id=2257. Accessed 25 Jan 2016

United Nations High Commissioner for Refugees (UNHCR) (2015a) Health services for Syrian refugees in Mount Lebanon and Beirut., http://data.unhcr.org/ syrianrefugees/download.php?id=8356. Accessed 25 Jan 2016 
United Nations High Commissioner for Refugees (UNHCR) (2015b) UNHCR Global Appeal 2015 Update., http://www.unhcr.org/5461 e607b.html. Accessed 25 Jan 2016

United Nations High Commissioner for Refugees (UNHCR) (2015c) Registration trends for Syrians in Lebanon., http://data.unhcr.org/syrianrefugees/ download.php?id=8585. Accessed 1 Oct 2015

United Nations Office for the Coordination of Humanitarian Affairs (OCHA) (2014) Lebanon Crisis Response Plan 2015-2016: Sector Plans., https://docs.unocha.org/sites/dms/CAP/2015-2016_Lebanon_CRP_Sector_ Plans_EN.pdf. Accessed 25 Jan 2016

World Bank (2013) Lebanon-economic and social impact assessment of the Syrian conflict., http://documents.worldbank.org/curated/en/2013/09/ 18292074/lebanon-economic-social-impact-assessment-syrian-conflict. Accessed 25 Jan 2016

World Health Organization (WHO) (2015) Lebanon: WHO Statistical Profile., http://www.who.int/gho/countries/lbn.pdf?ua=1. Accessed 25 Jan 2016

\section{Submit your manuscript to a SpringerOpen ${ }^{\odot}$ journal and benefit from:}

- Convenient online submission

- Rigorous peer review

- Immediate publication on acceptance

- Open access: articles freely available online

- High visibility within the field

- Retaining the copyright to your article

Submit your next manuscript at $\boldsymbol{s p r i n g e r o p e n . c o m ~}$ 